\title{
An Efficient Steganography with Mosaic Images for Covert Communication
}

\author{
Lisha . $\mathrm{L}^{1}$, Kavitha . $\mathrm{M}^{2}$ \\ ${ }^{1}$ (Department of Computer Science and Engineering, Jayamatha Engineering College-Nagarcoil) \\ ${ }^{2}$ ( Asst. Professor,Department of IT, Jayamatha Engineering College- Nagarcoil)
}

\begin{abstract}
The main aim of the paper is to analyze about security in image transfer through the network. Secretfragment-visible mosaic image is proposed for combining small tiles of secret image to form a target in the sense of mosaic. When this artwork is viewed at close, the observer can view smaller elements, yet when viewed at a distance the collection of tiles blend together to yield the overall picture. When the mosaic generating process starts, original image is divided into many tiles. Before splitting the image, compare the image for mosaic creation. Mosaic image is created automatically by composing small fragments of a given image in to target image, achieving an effect of embedding the given source image secretly in the resulting mosaic image. To create the mosaic image, first find the similar target image for selected source image. Finding the best fit tile for embedding in the target image blocks. The information of placing the tile image fitting sequence in target image is embedding into random selected pixel in created mosaic image. The embedded information act as a text key file. The key file contains the tile image fitting sequence. Without this key receiver can't retrieve the secret image.
\end{abstract}

Keywords: mosaic image, computer art, information hiding, covert communication.

\section{INTRODUCTION}

Steganography is the science of hiding secret messages into cover media so that no one can realize the existence of the secret data [1-2]. Existing steganography techniques may be classified into three categories image, video, and text steganographies, and image steganography aims to embed a secret message into a cover image with the yielded stego-image looking like the original cover image. Many image steganography techniques have been proposed, and some of them try to hide secret images behind other images.

Mosaic is a type of artwork created by composing small pieces of materials, such as stone, glass, tile, etc. Invented in ancient time, they are still used in many applications today. Creation of mosaic images by computer [1] is a new research direction in recent years.. A good survey under a unified framework can be found in Battiato et al. [2] in which a taxonomy of mosaic images into four types is proposed, including crystallization mosaic, ancient mosaic, photo-mosaic, and puzzle image mosaic. The first two types are obtained from decomposing a source image into tiles (with different colors, sizes, and rotations) and reconstructing the image by properly painting the tiles, and so they both may be called tile mosaics. The other two types of mosaics are obtained by fitting images from a database to cover an assigned source image, and both may be called multi-picture mosaics.

A new type of art image, called secret-fragment-visible mosaic image, which contains small fragments of a given source image is proposed in this study. Observing such a type of mosaic image, one can see all the fragments of the source image, but the fragments are so tiny in size and so random in position that the observer cannot figure out what the source image looks like. Therefore, the source image may be said to be secretly embedded in the resulting mosaic image, though the fragment pieces are all visible to the observer. And this is the reason why the resulting mosaic image is named secret-fragment-visible. which is the result of random rearrangement of the fragments of a secret image in disguise of another image called target image, creating exactly an effect of image steganography. The difficulty of hiding a huge volume of image data behind a cover image is solved automatically by this type of mosaic image. This is a new technique of information hiding, not found in the literature so far.

It is useful for the application of covert communication or secure keeping of secret images. Accordingly, we propose in this study a new method that creates secret-fragment visible mosaic images with no need of a database; we compare both secret and target image and find the most similar target image. .Fig. 2 shows a result yielded by the proposed method. Specifically, after a target image is selected, the given secret image is first divided into rectangular fragments, which then are fit into similar blocks in the target image according to a similarity criterion based on color variations. Next, the color characteristic of each tile image is transformed to be that of the corresponding block in the target image, resulting in a mosaic image which looks like the target image. Such a type of camouflage image can be used for securely keeping of a secret image in 
disguise of any pre-selected target image. Relevant schemes are also proposed to conduct nearly-lossless recovery of the original

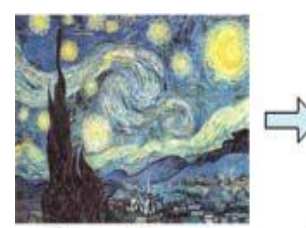

Secret image

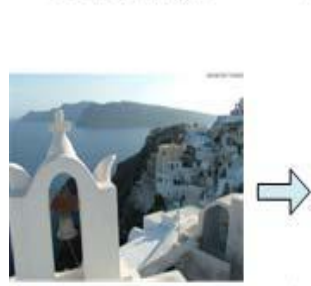

Target image

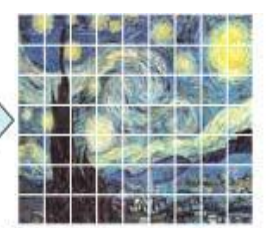

Divided secret image

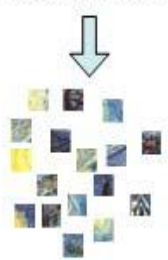
secret image.

Fig. 1. Illustration of creation of secret-fragment-visible mosaic image proposed

\section{RELATED WORK}

In traditional methods secret text can be hidden into image which is called as Steganography. In this method only text data can be encrypted but not image. Secret images can be hidden using water marking principles. Water marking is very simple process and it is weak that anyone can decrypt easily. Mosaic image technique is one of the efficient techniques to hide the secret images. This methodology needs another image which is said to be cover image. Creating mosaic image is also a art of computer. Many methods have been proposed to create different types of mosaic images by computer. Crystallization mosaic, ancient mosaic, photomosaic, and puzzle image mosaic are four types of mosaic images. An image is fragmented into small tiles. Then these tiles are randomly embedded onto a cover image. For encryption embedding process should be performed in some order. Secret key is used for embedding the small tiles of secret image onto cover image. LSB (least significant bit) replacement scheme is a technique mainly used for embedding process. LSB technique reduces or avoids the blur effect of encrypted mosaic image.

A novel approach based on Gradient Vector Flow (GVF) computation together with some smart heuristics used to drive tiles positioning. Almost all previous approaches filter out high frequencies in order to simplify mosaic generation. Preliminary works have shown that GVF properties permit us to preserve edge information and maintain image details. The novelty of this paper is related to the heuristics used to follow principal edges and to maximize the overall mosaic area covering. In particular the tiles positioning is not based only on gradient magnitude but makes use of local considerations to link together vectors that share the same "logical" edge. Experimental results confirm the better quality of the new technique with respect to the state of the art proposals.

Most of the reversible watermarking approaches proposed so far incorporate a lossless data compression stage. The use of an elaborate data compression stage increases the mathematical complexity of the watermarking. There are some watermarking schemes that do not rely on additional data compression, as for instance, the circular histogram interpretation schemes, but they have the drawback of a low embedding capacity. In this letter, we discuss a spatial domain reversible watermarking scheme that achieves high-capacity data embedding without any additional data compression stage. The scheme is based on the reversible contrast mapping (RCM), a simple integer transform defined on pairs of pixels. RCM is perfectly invertible, even if the least significant bits (LSBs) of the transformed pixels are lost. The data space occupied by the LSBs is suitable for data hiding. Here, a modified version that allows robustness against cropping is proposed. The control of distortions introduced by the watermarking is investigated as well. The mathematical complexity of the RCM watermarking is further analyzed, and a very low cost implementation is proposed. Finally, the RCM scheme is compared with Tian's difference expansion scheme with respect to the bit-rate hiding capacity and to the mathematical complexity.

Multimedia information retrieval (MIR) is about the search for knowledge in all its forms, everywhere. Indeed, what good is all the knowledge in the world if it is not possible to find anything? This sentiment is mirrored as an ACM SIGMM grand challenge [Rowe and Jain 2005]: "make capturing, storing, finding, and using digital media an everyday occurrence in our computing environment." This paper is meant for researchers in the area of content-based retrieval of multimedia. Currently, the fundamental problem has been how to enable or improve multimedia retrieval using content-based methods. Content-based methods are necessary when text annotations are nonexistent or incomplete. Furthermore, content-based methods can potentially improve retrieval accuracy even when text annotations are present by giving additional insight into the media collections. 
In ACM Transactions on Multimedia Computing, Communications, and Applications, Feb. 2006 Our search for digital knowledge began several decades ago when the idea of digitizing media was commonplace, but when books were still the primary medium for storing knowledge. Before the field of multimedia information retrieval coalesced into a scientific community, there were many contributory advances from a wide set of established scientific fields. From a theoretical perspective, areas such as artificial intelligence, optimization theory, computational vision, and pattern recognition contributed significantly to the underlying mathematical foundation of MIR. Psychology and related areas such as aesthetics and ergonomics provided basic foundations for the interaction with the user. Furthermore, applications of pictorial search into a database of imagery already existed in niche forms such as face recognition, robotic guidance, and character recognition.

\section{PROPOSED METHOD}

A flow diagram of the proposed method is shown in Fig. 1, which includes three phases of works:

Phase 1-creation of a secret-fragment-visible mosaic image using the tile images of a secret image and the selected similar target image as input;

Phase 2 -recovery of the secret image from the created secret-fragment-visible mosaic image.

The first phase includes three stages of operations:

Stage 1.1 - searching a target image most similar to the secret image;

Stage 1.2 -fitting the tile images in the secret image into the blocks of the target image.

Stage 1.3 -create a blank image to create a mosaic Image.

Stage 1.4-embedding the tile-image fitting information into the mosaic image for later secret image recovery.

And the second phase includes two stages of operations:

Stage 2.1 -retrieving the previously-embedded tile image fitting information from the mosaic image;

Stage 2.2 -reconstructing the secret image from the mosaic image using the retrieved information.

\section{A. Image similarity and target image selection}

The very first process of the project is picking or selecting most similar image for the source image. Selecting of target image is the important process, because of generating the mosaic for source image. In this, an efficient image similarity algorithm for comparison. Input for image similarity is the images and output of this is a percentage value for both secret and the target image. The value return from the algorithm determines how the similarity is. The input of image is given by the user only. If the input is text image or text file, he colored image is not suited. The second step is to split source image.

Split the source image into number of small piece of images called as tiles. The splitted image or tiles are placed with the target image for mosaic creation. Source splitting is directly propositional to the output quality. Source splitting size is high then output quality is very low, and split size is low then the quality of output is high.For example, split source image into $8 \times 8$ matrixes, finding the apt or correct position for placing the image in target is difficult. So that the quality is totally dropped in this size and the target image will get disappeared in this case. If split the image into 20x20 matrixes, quality is high .This will for the other similar splitting. Splitting of text image is also same as that of the colored image. But the output quality is totally differing from the quality of colored image. The image is splitted and ready for the further process to create the mosaic.

The purpose of going to this fixed square blocks is, the above methods are perfectly or suitable for generating the mosaic but not efficient retrieving process. For this disadvantage moving to the fixed square block. The simplest range partition consists of the fixed square blocks. Fixed block partitions, strongly neglect the image content. As a consequence, it leads to severe blocking artifacts and a rapidly deteriorating image quality. The coding concepts thus try to abandon fixed block partitions in favor of a content related picture representation. The usual solution is to introduce an adaptive partition with large blocks in low detail regions and small blocks where there is significant detail.

Let $\mathrm{Z} t$ be the size of each fragment. $\mathrm{N}$ number of tiles can be obtained.

$$
\mathrm{N}=\frac{\text { Ws X Hs }}{\mathrm{Zt}}
$$

Where Ws be the width of secret image, Hs be the height of secret image.

\section{B. Mosaic generation}

Before generating mosaic process, split target image into very small size. Here use separate splitting algorithm for split the target image and the size of the target image and tile is different. When the target image size is large can't able find the perfect match for tile. If it is small in size able to find the perfect match for each tile. The splitting of target image is finding the best fit for the tiles in the target image. The main problem behind 
this is there some replication of each image of tile and size of output image is too large compare to input image. Then compare the tile with all the splitted target images and get position of perfect match where the particular image is to be placed. After the finding of the best fit process, create a blank image with the back color is black.

The output image is made based on the position. Then get position of target image and place the tile into the blank black image. Concatenate output image will same as like that of target image. Here the placing will not overlap another image for that place image in a basis of tile image. Size of output image is not predicted earlier, output image size is based on the placing of the tile image. According to this the tile images will not overlap each other. The output image is with little quality drop lose but not more. The tile image size is too small then the computation time is high. The state of fitting a tile image is represented by a vertex of the graph, and the action of fitting the tile image into a target block may be represented by an edge of the graph with its weight taken to be the similarity value between the pixels' colors of the tile image and those of the target block. As said earlier the above process is for the text image, and here the target image is fully filled with tile images and background image is disappeared fully.

The state of fitting a tile image is represented by a vertex of the graph, and the action of fitting the tile image into a target block may be represented by an edge of the graph with its weight taken to be the similarity value between the pixel colors of the tile image and those of the target block.File sequence is the information file that consists of data of where the tile is placed in target image. While in creation of mosaic process best fit of particular tile is taken and stored in text file for future use. Hence the mosaic image didn't contain any information of stored image in it and it has only the image of tile. For construct the final output are need a position file to make the output as input. For this purpose use the merging information file. As considered above this contains where were the tile images are stored and name of the image is stored in particular position are available in this file. Embedding file consist of merging information. This file would be combined or merged with output image and send it to sender without any loss. There is a security transaction is held between the sender and the receiver. Without a de-embedding algorithm the others can't read the file or they can't separate the file. For this security a highly secure method is used for embedded the both files. After the completion of the embedding process the output will ready to send to the receiver.

Repetitively select randomly a block in unselected so far other than the first block of using the random number generator with the secret key as the seed, and embed bits of into all the pixels of by the lossless LSB replacement scheme proposed in until all the bits in are exhausted, where is computed Take the final with embedded as the desired secret-fragment-visible mosaic image for the input secret image and exit. A different technology is hiding the fitting sequence file into created mosaic image. The created mosaic image is converted to string for hiding the fitting sequence information file. After getting the sequence file, separates the string by an order for information hiding. Here the each and every line in the file is added to the converted string image file. The order is after every 25 characters the line of the fitting sequence file added. This will happen until added the entire line in the fitting sequence file.

\section{Reconstruction of secret image}

This is the final step of the project; the output is same as the input in sender side. Before going to reconstructing first de-embedded the image file and the text file. After that do the creation process in reverse process. De-embedded the file first and then split the mosaic created output image with the basis of text file and named the image as represented in text file. There are two steps us follows. The retrieve process is based on width and height of tile image in mosaic creation process. Split the mosaic image with duplicates as belong to size of tile and named it as name in position file.

In text file the position are not in the same order (i.e.) here the position file has the name corresponding to the image. Repetitively select randomly an unselected block other than the first block from using the random number generator with the secret key as the seed, extract bits from all the pixels of using a reverse version of the lossless LSB replacement scheme proposed in and concatenate them sequentially, until all the bits of are extracted. Transform every bits of into an integer which specifies the index of a tile image in the original secret image (to be composed), resulting in the secret recovery sequence. After getting the tile images from position file then there is no replication of images because the image is repeated then the name would be same and overwrite the previous image.

\section{EXPERIMENTAL RESULTS}

The processing time and quality is based on the tile image size. Split the source image into number of small piece of images called as tiles. If the tile size is small then the output mosaic image quality is high .For example, split source image into $8 \times 8$ matrixes, finding the apt or correct position for placing the image in target is difficult. So that the quality is totally dropped in this size and the target image will get disappeared in this case. If split the image into $20 \times 20$ matrixes, quality is high. 


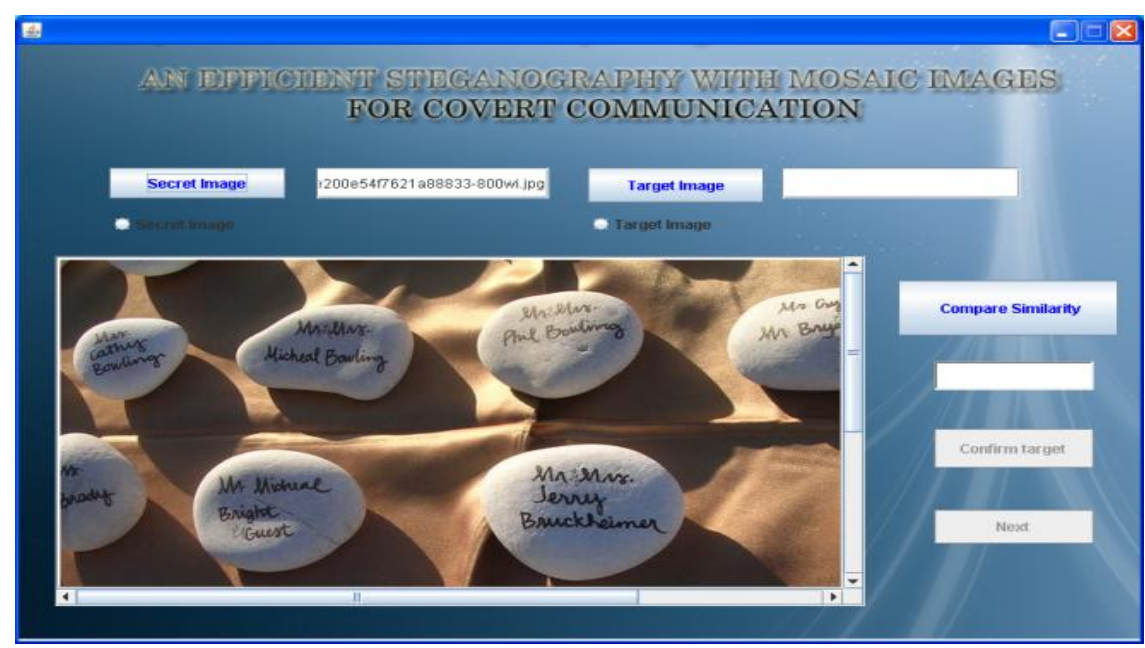

Fig. 2. Select the secret image

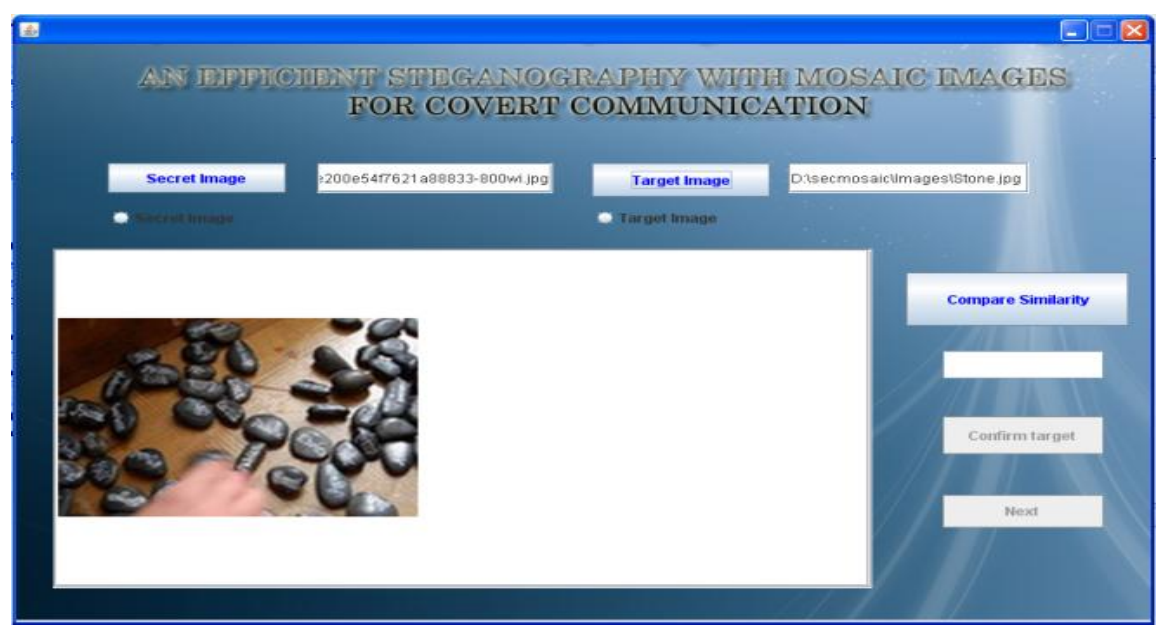

Fig. 3. Select the target image

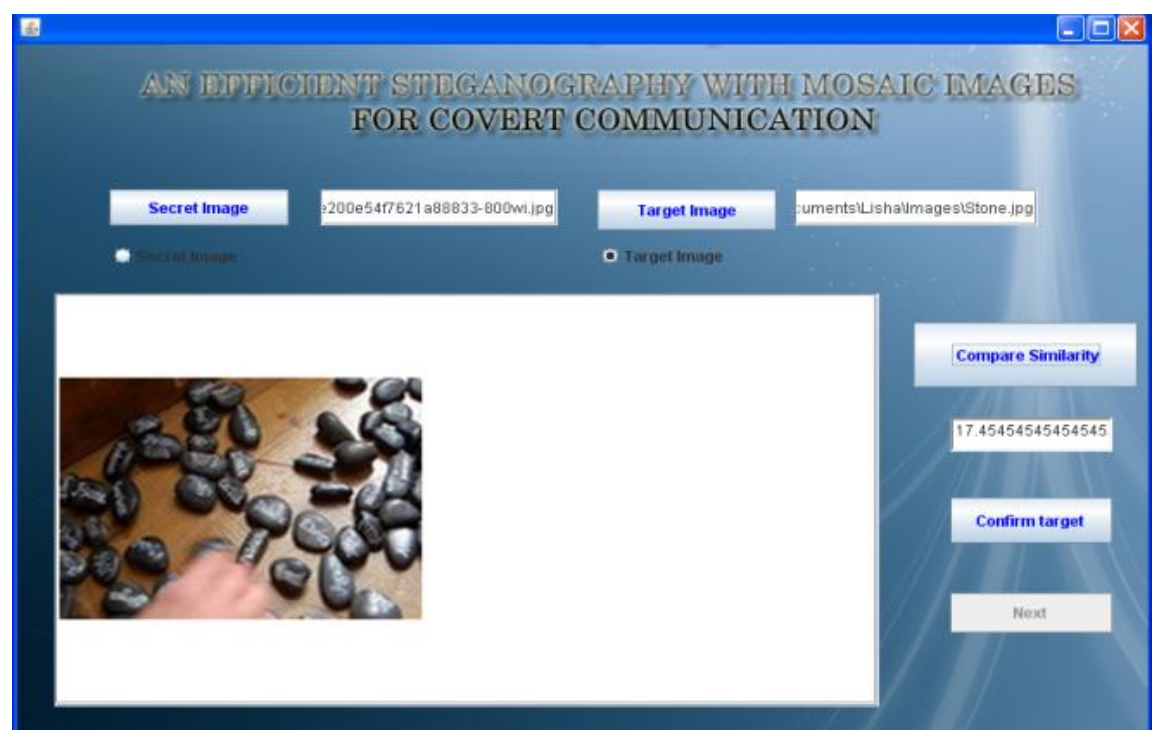

Fig. 4. Compare the similarity. 


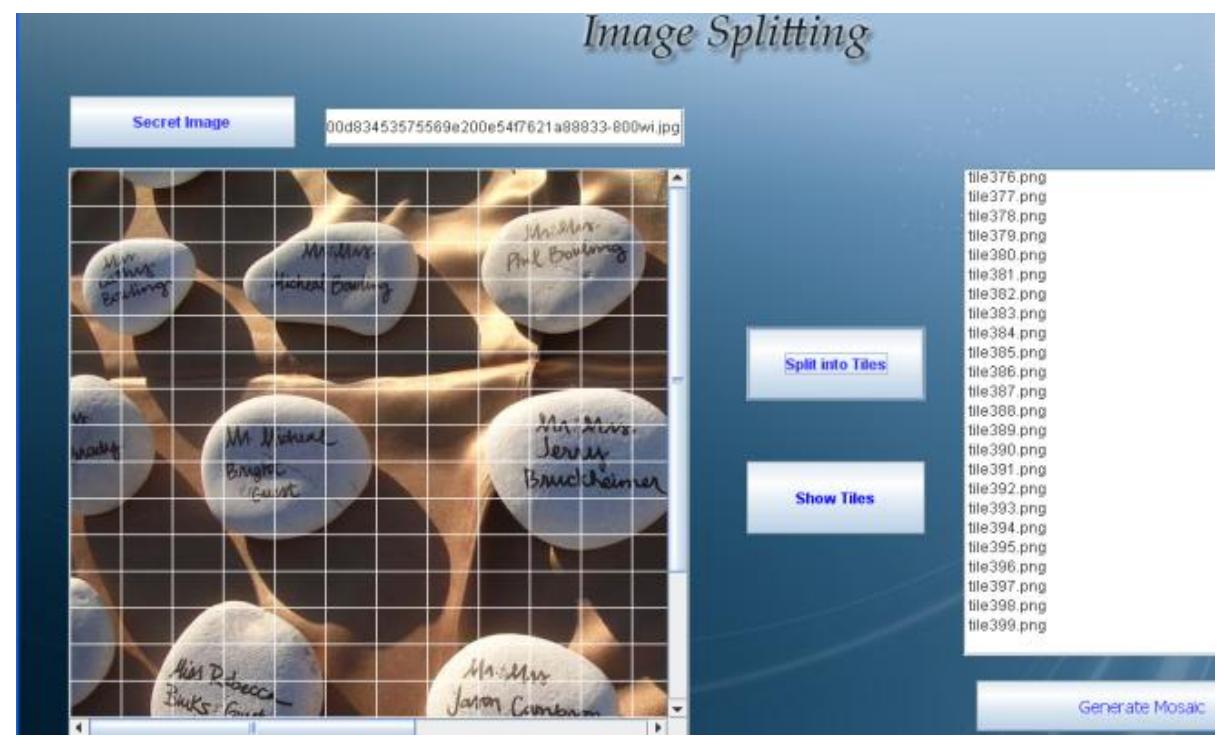

Fig. 5. Split the secret image

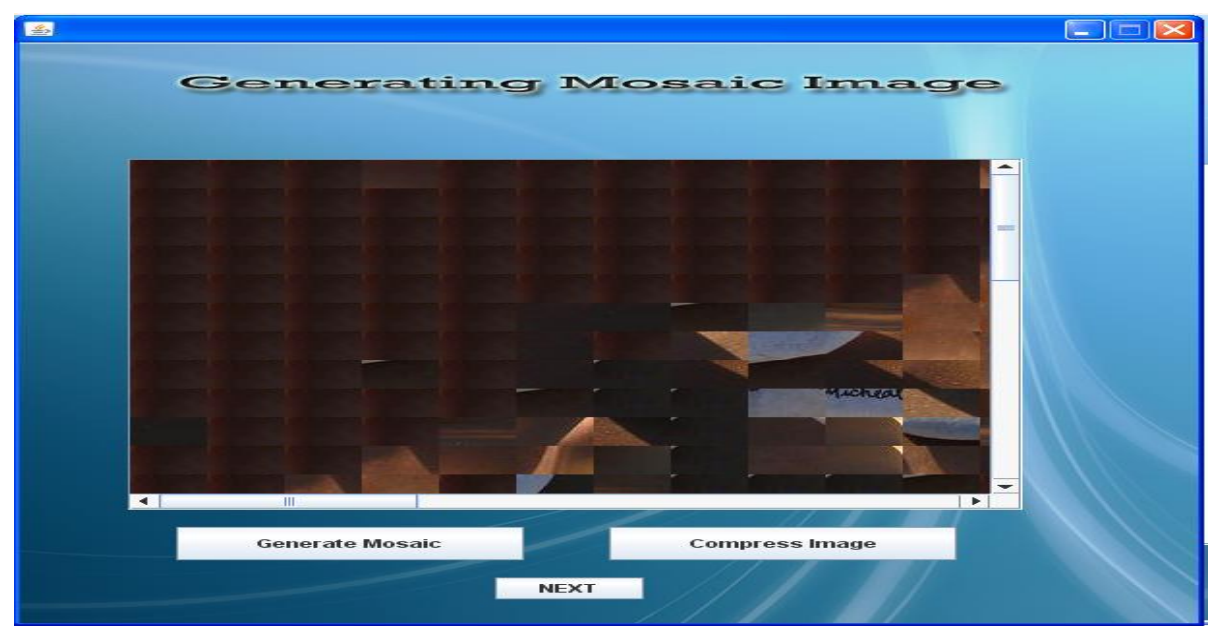

Fig. 6. Creation of mosaic

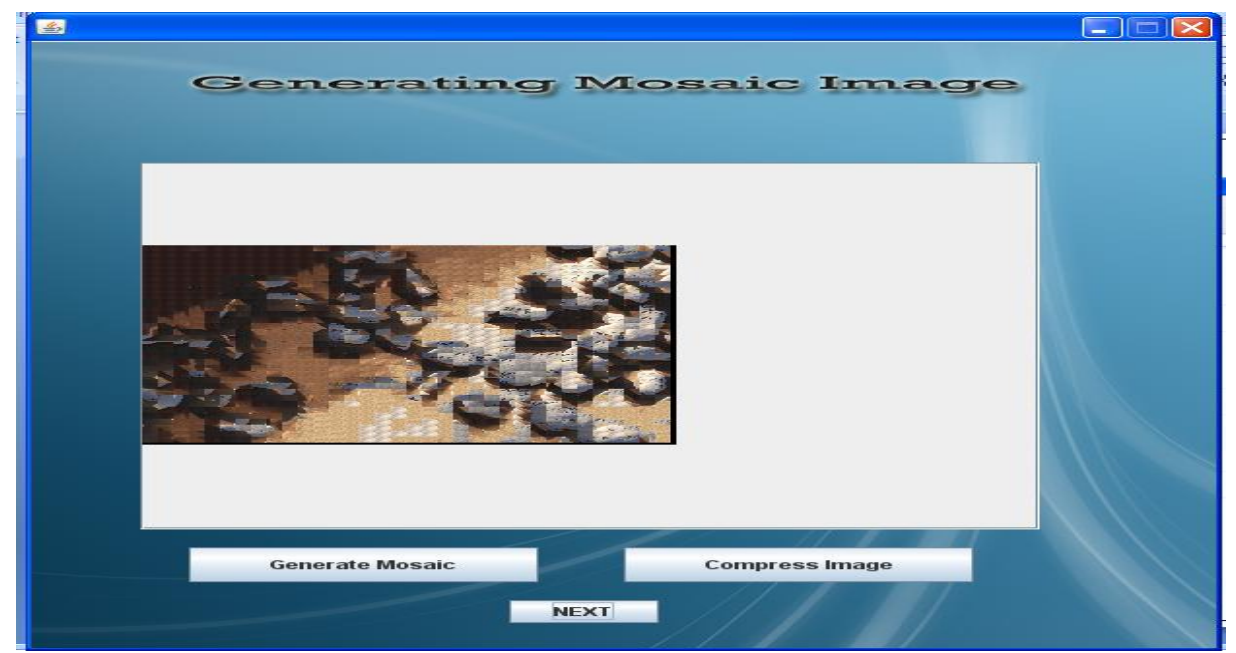

Fig. 7. Compression of mosaic 


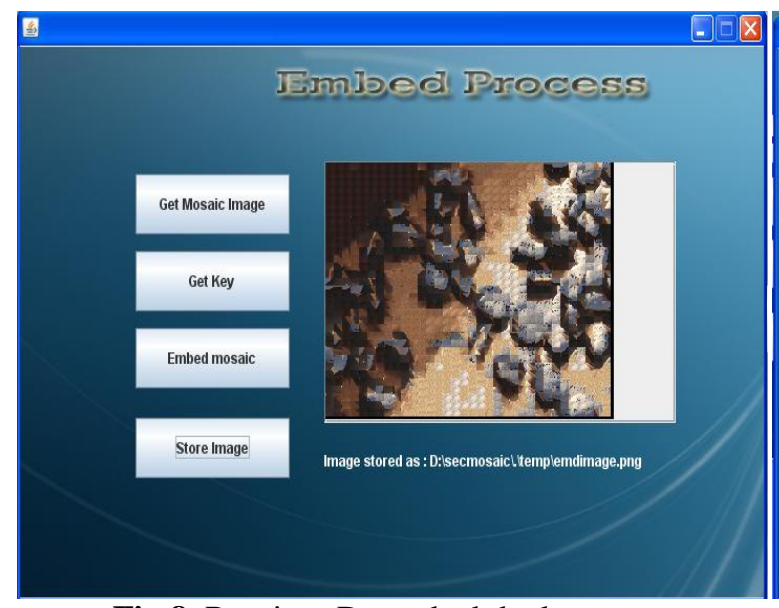

Fig.8. Receiver De-embed the key

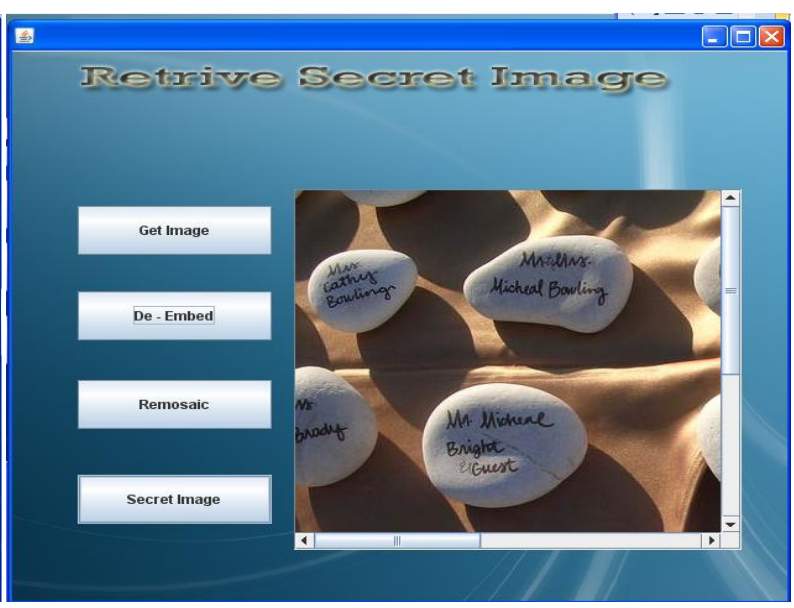

Fig. 9. Receiver retrieves the secret image

\section{CONCLUSION}

The new art which can be used for secure keeping or covert communication of secret images. This type of mosaic image is composed of small fragments of an input secret image and though all the fragments of the secret image can be seen clearly, they are so tiny in size and so random in position that people cannot figure out what the source secret image looks like. A novel algorithm has also been proposed for searching the tile images in a secret image for the most similar ones to fit the target blocks of a selected target image more efficiently.

\section{REFERENCES}

[1] M. K. Agoston, "Computer Graphics and Geometric Modeling: Implementation and Algorithms.” New York: Springer-Verlag, 2004.p.61.

[2] S. Battiato and "3D ancient mosaics," Proceedings of ACM International Conference on Multimedia (ACM Multimedia 2010) - Technical Demo, Florence, Italy, Oct. 2010, p 1571-1574.

[3] S. Battiato, G. Di Blasi, G. Gallo, G. C. Guarnera, and G. Puglisi, “Artificial mosaic by gradient vector flow," in Proc. Eurographics,Creete, Greece, Apr. 2008, pp. 53-56.

[4] Y.-S.Choi, B.-k.Koo, and B.-R.Moon, "Optimization of an image set by genetic feature selection for realtime photomosaics,” in Proc. GECCO, Portland, OR, Jul. 2010, pp. 1309-1310.

[5] D. Coltuc and J. M. Chassery, "Very fast watermarking by Reversible Contrast Mapping," IEEE Signal Process. Lett., Apr. 2007 vol. 14, no. 4, pp.255-258,.

[6] G. Di Blasi, G.M.Farinella, S.Battiato, and G. Gallo, "Digital mosaic framework: An overview," Eurograp Comput. Graph. Forum, , Dec. 2007,vol. 26, no. 4, pp. 794-812.

[7] Y.Dobashi, T.Haga, H.Johan, and T. Nishita, "A method for creating mosaic image using voronoi diagrams," in Proc. Eurographics,Saar-brucken, Germany, Sep. 2002, pp. 341-348.

[8] .DiBlasi and G.Gallo, “Artificial mosaics,” Vis. Comput.,April 2005, vol. 21, pp. 373-383,

[9] G.Elberand, G.Wolberg "Rendering traditional mosaics,” Vis. Comput.,june 2003 vol. 19, pp. 67-78,

[10] C.Guarnera,S.Battiato,G.DiBlasi,G.Gallo, and G.Puglisi ,M. Bubak, Ed. et al., "A novel artificial mosaic generation technique driven by local gradient analysis," in Proc. ICCS, Crakov, Poland, Jun. 2008, vol. 5102, pp. 76-85..

[11] A.Hausner, "Simulating decorative mosaics," in Proc. SIGGRAPH, Los Angeles, CA, Aug. 2001, pp. 573-580.

[12] J.Kim and F.Pellacini, "Jigsaw image mosaics," in Proc. SIGGRAPH, San Antonio, TX, Jul. 2002, pp. 657-664.

[13] M. S. Lew, N. Sebe, C. Djeraba, and R. Jain, "Content-based multimedia information retrieval: State of the art and challenges," ACM Trans. Multimedia Comput., Commun., Appl., pp. 1-19, Feb. 2006.

[14] Petralia G.DiBlasi, and M G.Gallo, , "Puzzle image mosaic," in Proc. IASTED/VIIP, Benidorm, Spain, Sep. 2005, pp. 33-37.

[15] R.Silverand,M.Hawley, Photomosaics. New York: Henry Holt, 1997, v.57.

[16] Vijay Kumar Sharma ,Vishal Shrivastava "A Steganography Algorithm For Hiding Image In Image By Improved LSB Substitution By Minimize Detection.” Journal of Theoretical and Applied Information Technology April 2007, vol 36. 